\title{
Effects of Nonlinearity on the Angular Drift Error of an Electrostatic MEMS Rate Integrating Gyroscope
}

\author{
Zhongxu Hu, Barry Gallacher \\ School of Mechanical and System Engineering, Newcastle University, Newcastle upon Tyne, NE1 7RU, UK (e-mail: Zhongxu.hu@ncl.ac.uk).
}

\begin{abstract}
Electrostatic MEMS Coriolis vibratory gyroscopes (CVG) are essentially nonlinear because of the capacitive transducers employed for the excitation and detection of resonance vibration. This paper investigates the influence of nonlinearity on the precession angle dependent bias error of a MEMS rate integrating gyroscope (RIG) and proposes a novel correction to minimize this effect. A linear model of CVGs is commonly used in the dynamic analysis and control of MEMS RIGs. The linear model predicts a $2^{\text {nd }}$ harmonic angular drift error [1][2] due mainly to non-proportional damping. However, experimental results from previous work [3][4] demonstrate the existence of an additional $4^{\text {th }}$ harmonic component in the precession rate, as well as in the resonant frequency and quadrature control. Analysis and removal of this high order error term will further improve the accuracy of RIG. Here, it is shown that high order angularly modulated drift error is the result of nonlinear damping, and that the stiffness nonlinearity is responsible for the $4^{\text {th }}$ harmonics present in the fluctuation of the operating frequency and in the control for quadrature nulling. It is understood that nonlinear damping may be introduced electrically by the energy sustain state feedback control that uses nonlinear capacitive measurements. Nonlinearity correction is proposed to the capacitive displacement detection which significantly reduces the high order drift error. Simulation and experimental results are provided to validate the analysis. A DSP controlled MEMS RIG with nonlinearity correction exhibits an angular drift error of less than $0.2 \mathrm{deg} / \mathrm{s}$.
\end{abstract}

Index Terms - Rate Integrating Gyroscope; Electrostatic nonlinearity; Low angular drift; Nonlinearity correction;

\section{INTRODUCTION}

$\mathrm{M}$ EMS Coriolis vibratory gyroscopes $(\mathrm{CVG})$ are essential components in compact and low cost inertial applications, for example, the attitude and heading reference system (AHRS) in UAVs, personal navigation and robotics. The working principle of a $\mathrm{CVG}$ is based on the Coriolis coupling induced energy transfer between a pair of degenerate vibration modes associated with axisymmetric structures e.g ring, cylinder, disk and hemispherical shell. Most CVGs are operated in rate mode, where measured rate of turn is integrated to obtain the angular position. Due to the fact that the rate measurements are often affected by the temperature induced bias instability, nonlinearity in the scale factor, noise and limited measurement bandwidth, the derived angle can diverge over a rather short period of integration time. High end CVGs can be operated in rate integrating mode that directly give precession angle without the need of digital integration.
Other advantages of RIGs include: (1), scale factor stability unaffected by environmental changes and amplitude of vibration of the drive mode; (2), unlimited bandwidth and measurement range, though in practice, this benefit may be compromised by bandwidth of the electronic control loops. Despite the attractive advantages of RIGs, their closed-loop control is much more challenging and it is only possible for gyroscope devices with minimum imperfections [5][6]. The major performance specifications for RIGs are the precession angle dependent bias error and the minimum rate threshold. Drift error is the major limiting factor for MEMS RIGs in low rate applications. They are mainly affected by damping and stiffness imperfections caused by fabrication tolerance limits and material inhomogeneity. Gain unbalance in drive and sense electronics, and the phase lag of actual control forces also contribute to drift error.

Most of the recent works [7][8] conducted on MEMS RIGs have adopted the linear dynamic model in the design of control algorithms and for the analysis of drift error. The averaged motion equations based on the linear dynamic model of CVGs reveal that the damping and stiffness imperfections between the two resonators are the major sources of angular drift error. The influence of stiffness imperfections can be eliminated conveniently by quadrature nulling which keeps the resultant vibration mode shape a straight line. The drift error caused by non-proportional damping can be compensated via velocity feedback control [7][8]. However, the linear model-based analysis only explains the existence of a secondary spatial harmonic in the drift error. Previous works [3][4] by the authors show that, as the dominant $2^{\text {nd }}$ harmonic is reduced, there exists a $4^{\text {th }}$ spatial harmonic component present in the drift error, quadrature nulling control and resonance frequency. Further tests show that the state feedback compensation techniques only reduce the $2^{\text {nd }}$ harmonic and have no effect on the high order error component. This implies that the $4^{\text {th }}$ harmonic error may be due to the nonlinear damping and stiffness contributions which are commonly neglected in the linear model of MEMS CVGs. As the $2^{\text {nd }}$ harmonic in the angular drift error can be effectively eliminated via state feedback compensation, removal of the $4^{\text {th }}$ harmonic drift error represents a significant advancement.

Elastic and dissipative nonlinearities exist in all MEMS resonators. For electrostatic MEMS gyroscopes, nonlinearity may originate from several sources including the geometric and material effects, electrostatic actuation and capacitive detection. Nonlinear behavior and bifurcation of MEMS 
resonators caused by elastic and damping nonlinearities are extensively reported [9][10][11][12]. Most of these studies are based on simple 1D clamped-clamped microscale [13] and nanoscale [14] beam resonators. One of the most reported phenomena is the amplitude-frequency dependence caused by cubic stiffness nonlinearity. Damping nonlinearity manifests as a reduced apparent quality factor as the amplitude of vibration increases. Novel methods [15] [16] are recently used to reduce drift error in MEMS RIGs. The influence of cubic stiffness nonlinearity on the performance of MEMS rate gyroscopes is investigated [17][18][19][20], where the amplitude of vibration of the primary mode is pushed well into the nonlinear region. Effects of this include the proportionally amplified scale factor, improved signal to noise ratio performance. The bias stability is generally unaffected.

In contrast to the intensively investigated nonlinear elastic behavior, nonlinear damping in MEMS gyroscopes is often overlooked as its influence on the rate gyro is negligible for most applications. Damping nonlinearity is much more apparent when the gyro is operating in rate integrating mode. It is very challenging to quantitatively identify mechanical nonlinearities. In vacuum packaged MEMS gyroscopes, thermal elastic damping (TED) is an important contributor. Explicit analyses of the effect of elastic and dissipative nonlinearities on the measurement performance of RIGs are rare or incomplete. In this paper the mechanism by which nonlinearity is introduced to the RIG system through feedback control is analysed. The influence of nonlinearity on the drift error performance of a MEMS RIG is assessed. Correction of nonlinearities from electrostatic sensing is proposed and validated via simulation and experimentation. The gyroscope device used in this study is a ring resonator of radius $4 \mathrm{~mm}$ and a capacitive gap of $10 \mu \mathrm{m}$. It has a quality factor of 22000 due to extremely low damping, and the amplitude of vibration is kept low in the linear region, so that the geometrical and material nonlinearities are negligible for this design when compared against the nonlinearity stemming from the electrostatic transduction.

The outline of the paper is as follows. Section 2 introduces the dynamic model of CVGs, nonlinearities originating from electrostatic forces and the capacitive detection scheme, and the mechanism these nonlinearities are introduced into the gyroscope control system via state feedback control. Section 3 describes the derived averaged motion equations of the RIG with damping and stiffness nonlinearities. A theoretical analysis of their effects on the angular drift error and associated control are also presented. Section 4 presents a SIMULINK based simulation of the entire nonlinear RIG control system which concurs with the theoretical analysis. Practical rate table tests are conducted with a DSP controlled fully functioning MEMS RIG. The implementation of the DSP based control system and test results are given in section 5 to show the effectiveness of nonlinearity correction in reducing high order drift error.

\section{NONLINEAR DYNAMIC MODEL}

A Coriolis vibratory gyroscope is commonly modeled as a twodimensional oscillator with damping and stiffness couplings. Without considering stiffness and damping nonlinearities from mechanical sources, the mass normalized differential equations of motion are described as [18]:

$$
\begin{aligned}
& {\left[\begin{array}{l}
\ddot{x} \\
\ddot{y}
\end{array}\right]+\left\{2 \vartheta_{0} \omega_{0}\left[\begin{array}{cc}
1+\gamma_{1} & \gamma_{2} \\
\gamma_{2} & 1-\gamma_{1}
\end{array}\right]+\left[\begin{array}{cc}
0 & -2 \Omega \\
2 \Omega & 0
\end{array}\right]\right\}\left[\begin{array}{l}
\dot{x} \\
\dot{y}
\end{array}\right]+} \\
& \omega_{0}{ }^{2}\left[\begin{array}{cc}
1+\mu_{1} & \mu_{2} \\
\mu_{2} & 1-\mu_{1}
\end{array}\right]\left[\begin{array}{l}
x \\
y
\end{array}\right]=\left[\begin{array}{l}
F_{x} \\
F_{y}
\end{array}\right]
\end{aligned}
$$

Where $x$ and $y$ are the displacement of vibration, $\vartheta_{0}$ and $\omega_{0}$ are respectively the damping ratio and natural frequency of the perfect ring resonator. $\gamma_{1}, \gamma_{2}$ and $\mu_{1}, \mu_{2}$ represent the damping and stiffness imperfections respectively. $\Omega$ is the external rate input that induces the Coriolis coupling, which is proportional to the velocity of vibration and corresponding mass of the resonator $m . F_{x}=\frac{k_{d} V_{x}}{m}$ and $F_{y}=\frac{k_{d} V_{y}}{m}$ are electrostatic forces applied to the two modes of vibration by individual control voltages $V_{x}$ and $V_{y}$, and $m$ is the resonator mass. In the case of the RIG mode of operation, the objective of feedback control is to sustain vibration in the major axis and suppress vibration in the minor axis in the elliptic coordinate system [2]. The electrostatic force coefficient $k_{d}$ is determined by the capacitive gap and the DC bias.

\section{A. Nonlinear Electrostatic Force}

Nonlinearity in high Q MEMS resonators may originate from both mechanical and electrical sources. Electrostatic forces and the capacitive detection of displacement are naturally nonlinear and appear as a softening nonlinearity that decrease the resonant frequency as shown in (5) and (6). Mechanical nonlinearity, commonly modelled as a duffing force $\alpha x^{3}$, has a spring hardening characteristic that increases the effective stiffness at large displacements. The combination of these nonlinearities can be hardening or softening depending on the strength of these two nonlinearities. For the MEMS ring type gyroscope used in this study, the ring resonator has a radius of $4 \mathrm{~mm}$, and the amplitude of vibration is in the range of a couple of micrometers over a capacitive gap of $10 \mu \mathrm{m}$. Geometric nonlinearity caused by this small amplitude of vibration relative to the dimension of the ring resonator is negligible. Electrostatic transduction is major source of stiffness nonlinearity and can be readily confirmed by simple frequency sweep tests, as shown in figure (1). The resonant frequency shifts $1.5 \mathrm{~Hz}$ lower as the amplitude of vibration increases.
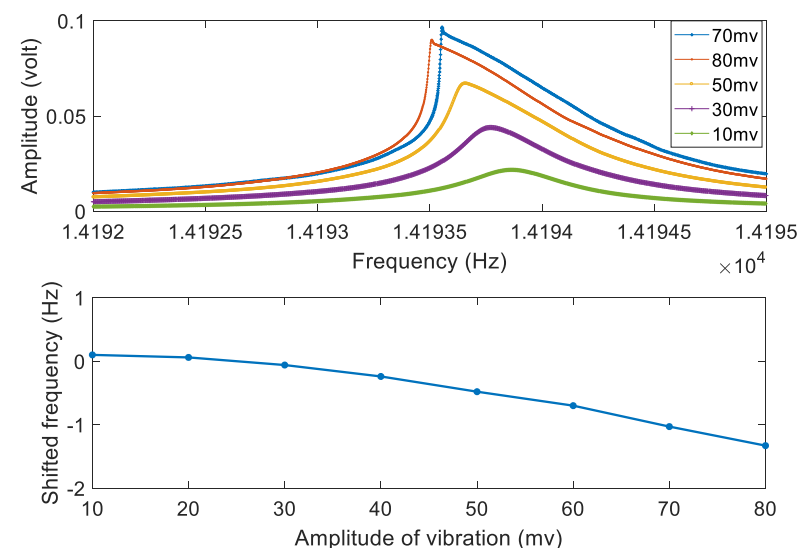

Fig.1. Frequency response shows softening nonlinearity dominated by 
electrostatic effects.

As the radius of the ring resonator is large compared with the nominal capacitive gap, stiffness nonlinearity in electrostatic actuation can be modelled based on parallel plate electrodes. The attractive electrostatic force produced between the ring resonator body and the drive electrode is expressed as a nonlinear function of amplitude of vibration:

$$
F_{e}=\frac{\varepsilon A}{2(d-x)^{2}}\left(V_{d c}-V_{a c}\right)^{2}
$$

Where $d$ is the nominal gap between the ring resonator body and the drive electrode, $x$ is the displacement, $V_{d c}$ is the DC bias of 25 volts, $V_{a c}$ is the periodic drive voltage, and $V_{a c} \ll$ $V_{d c}$. The reason of using a fixed high DC bias $V_{d c}$ and a low amplitude alternative voltage $V_{a c}$ for harmonic excitation is that it avoids nonlinearity caused by the voltage square term. Using Taylor expansion, and neglecting high order terms, the electrostatic force can be approximately expressed as:

$$
F_{e} \cong \frac{C_{0}}{2 d}\left(V_{d c}-V_{a c}\right)^{2}\left[1+2 \frac{x}{d}+3\left(\frac{x}{d}\right)^{2}+4\left(\frac{x}{d}\right)^{3}\right]
$$

Here $C_{0}$ is the nominal capacitance of the gap. The first term in equation (3) is the linear harmonic excitation force independent of displacement of vibration. It is linearly proportional to the alternative drive voltage $V_{a c}$, approximately given by

$$
F_{e l}=k_{d} V_{a c}
$$

$k_{d}=\frac{C_{0} V_{d c}}{d}$ is the coefficient for the linear electrostatic force, which is dependent on the DC bias and the electrode gap. The displacement linear, quadratic and cubic terms of the spring softening forces are given by

$$
F_{e s}=\frac{C_{0} V_{d c^{2}}}{d^{2}}\left[1+\frac{3}{2}\left(\frac{x}{d}\right)+2\left(\frac{x}{d}\right)^{2}\right] x
$$

As the DC bias voltage is fixed the linear term in (5) of stiffness softening remains constant despite varying amplitude of harmonic drive $V_{a c}$ and amplitude of vibration. The quadratic and cubic nonlinearity terms are related to the amplitude of vibration, which will result in frequency mismatch in RIG mode where the amplitude of vibrations for the two modes vary alternatively with precession angle. As the quadratic term introduces a high frequency modulation to the effective stiffness, the averaged effect on the slow time-varying averaged motion equations of RIG envelope dynamics is negligible. Therefore, only the cubic nonlinearity term of the electrostatic force is considered later in the analysis of the nonlinear dynamic system of RIG.

\section{B. Nonlinearity in Capacitive Detection}

The sensing electrode and the ring resonator form a DC biased capacitor modulated by displacement of vibration. A charge amplifier is used in the capacitive detection of vibration, the voltage output of detection circuit $V_{\text {out }}$ is given by

$$
V_{\text {out }}=\frac{1}{C_{F}} \frac{\varepsilon A}{d-x} V_{d c} \approx \frac{C_{0}}{C_{F}} V_{d c}\left[1+\left(\frac{x}{d}\right)+\left(\frac{x}{d}\right)^{2}+\left(\frac{x}{d}\right)^{3}\right]
$$

$C_{F}$ is feedback capacitor of the charge amplifier. To avoid the electrostatic instability phenomena of "pull-in" the amplitude of vibration is kept small such that $x \ll d$. As a result, terms $O\left(\frac{x}{d}\right)^{n}$ for $n>3$ have been neglected in expression (6). Furthermore, a high pass filter in the interface electronics removes the DC component in $V_{\text {out }}$ such that the output signal $V_{S}$ is given by

$$
V_{s} \cong \frac{C_{0}}{C_{F} d} V_{d c}\left[1+\frac{x}{d}+\left(\frac{x}{d}\right)^{2}\right] x
$$

Note that the signal described by (7) is not purely proportional to the amplitude of vibration. Neglecting high order harmonic vibrations that may be caused by dynamic nonlinearities, the displacement of vibration may be expressed as a pure sinusoidal function $x=x_{p} \sin \omega t$. The corresponding output signal of the charge amplifier can be written as [12]:

$$
\begin{aligned}
V_{s} \cong & \frac{C_{0}}{C_{F} d} V_{d c}\left[\left(1+\frac{3}{4}\left(\frac{x_{p}}{d}\right)^{2}\right) x_{p} \sin \omega t-\frac{x_{p}^{2}}{2 d} \cos 2 \omega t-\right. \\
& \left.\left(\frac{x_{p}}{2 d}\right)^{2} x_{p} \sin 3 \omega t\right]
\end{aligned}
$$

The relative amplitude of the spectrum components in the detected vibration signal may be used to estimate the true displacement relative to the gap $d$. The harmonic components $2 \omega$ and $3 \omega$ in (8) are removed by the synchronous demodulation process from entering the closed-loop feedback control system. The effective signal entering the RIG control system is given by:

$$
V_{s} \cong \frac{C_{0}}{C_{F} d} V_{d c}\left[1+\frac{3}{4}\left(\frac{x_{p}}{d}\right)^{2}\right] x
$$

It can be seen from (9) that the displacement of vibration becomes over-estimated as the amplitude of vibration increases. This can be problematic for rate integrating gyroscope where the amplitude of vibration of both modes changes alternatively during angular precession. In parallel plate electrostatically actuated MEMS resonators the maximum displacement before the onset of pull-in stability is given by $x_{M} \cong \frac{d}{3}$. In practice the resonator is operated with $x \ll x_{M}$. Nonlinearity originating from capacitive detection enters into the system dynamics through quadrature nulling and energy sustaining feedback control, which will introduce both stiffness and damping nonlinearities

\section{Nonlinear Damping}

Nonlinear damping in high Q MEMS gyroscopes is often neglected in rate mode CVGs. However, in the RIG mode, it may cause extra angular drift error. Nonlinear damping may originate from a combination of different sources, including air damping, thermoelastic effects, friction at grain boundaries, bulk and surface impurities and clamping loss. It is not possible to identify and estimate these sources individually. A cubic nonlinear damping force is often introduced to model the damping nonlinearity [13][14]. Another major source of nonlinearity may come from the quadrature nulling and energy sustaining closed-loop controls. The cubic nonlinearity in the 
capacitive displacement detection scheme described by equation (6) is introduced to the full dynamic system of the RIG.

In the implementation of closed-loop feedback control, control voltages $V_{x}$ and $V_{y}$ are synthesized using state variables $\hat{x}, \dot{\hat{x}}, \hat{y}, \dot{\hat{y}}$ measured by the capacitive detection circuits. The quadratic term in (6) is prevented from entering the control system by the synchronous demodulation. The measured state variables are given by:

$$
\begin{aligned}
& \hat{x} \cong k_{s}\left[1+\left(\frac{x}{d}\right)^{2}\right] x, \quad \dot{\hat{x}} \cong k_{s}\left[1+3\left(\frac{x}{d}\right)^{2}\right] \dot{x} \\
& \hat{y} \cong k_{s}\left[1+\left(\frac{y}{d}\right)^{2}\right] y, \quad \dot{\hat{y}} \cong k_{s}\left[1+3\left(\frac{y}{d}\right)^{2}\right] \dot{y}
\end{aligned}
$$

$k_{s}=\frac{C_{0}}{C_{F} d} V_{d c}$ is the gain of the electronic circuit, which is dependent on the capacitive gap, the feedback capacitor and the DC bias. The conventional feedback controls for quadrature nulling and energy sustain [3][8] are given by:

$$
\begin{array}{r}
V_{x}=\bar{\alpha} \hat{y}+\bar{\beta} \dot{\hat{x}} \\
V_{y}=-\bar{\alpha} \hat{x}+\bar{\beta} \dot{\hat{y}}
\end{array}
$$

Here, nonlinearities caused by feedback compensation of the linear non-proportional damping [3] $\gamma_{1}, \gamma_{2}$ are neglected, as the non-proportional damping terms are small relative to the average damping. $\bar{\alpha}$ and $\bar{\beta}$ are control gains for the quadrature nulling and energy sustain loops respectively. This effectively introduces cross stiffness nonlinearity and diagonal damping nonlinearity terms to the dynamics of RIG by electrostatic feedback forces. The mass normalized forces for the gyroscope dynamic model (1) are given by:

$$
\begin{aligned}
& F_{x}=k_{3} x^{3}+\frac{\alpha}{d^{2}} y^{3}+\frac{3 \beta}{d^{2}} x^{2} \dot{x}+\alpha y+\beta \dot{x} \\
& F_{y}=k_{3} y^{3}-\frac{\alpha}{d^{2}} x^{3}+\frac{3 \beta}{d^{2}} y^{2} \dot{y}-\alpha x+\beta \dot{y}
\end{aligned}
$$

Here, $k_{3}=\frac{2 C_{0} V_{d c}{ }^{2}}{d^{4} m}, \alpha=\frac{\bar{\alpha} k_{d} k_{s}}{m}$, and $\beta=\frac{\bar{\beta} k_{d} k_{s}}{m}$. Equations (14) (15) show the stiffness and damping nonlinearities introduced by electrostatic drive and capacitive sensing. Nonlinear damping from geometrical and material effects are often approximately modelled as $r_{3} x^{2} \dot{x}, r_{3} y^{2} \dot{y}$, which can be conveniently included in (14) (15). However, in this investigation, as the amplitude of vibration is very small relative to the radius of the ring resonator, nonlinear damping arising from geometrical and material aspects is neglected. Nonlinear damping introduced by feedback control forces due to distortion in the capacitive detection is the major source. Nonlinear damping from mechanical and material sources is often characterized as decreased Q factor as the amplitude of vibration is increased, as has been found in simple 1-D doubly clamped M(N)EMS structures [13][14]. Rather differently, nonlinear damping introduced by feedback control in electrostatic MEMS devices reduces effective damping of the resonant modes and hence increases the observed $\mathrm{Q}$ factor.

\section{Nonlinearity Correction}

As described in equation (10), the nonlinear error in the capacitive detection is related to the ratio of displacement and the nominal capacitive gap. A simple correction to the nonlinear measurement is proposed by subtracting the cubic term from the measurement,

$$
x=\hat{x}-k_{s} \frac{3}{4}\left(\frac{|x|}{d}\right)^{2} x
$$

An approximation to the unknown true displacement $x$ in the correction equation (16) can be made using the linear part of the measurement result $x=\hat{x} / k_{s}$. The nonlinear error can be reduced significantly provided parameters $k_{S} d=V_{d c} \frac{C_{0}}{C_{F}}$ or the real amplitude of vibration relative to the capacitive gap $d$ are exactly known. The corrected measurement result is given by

$\tilde{x}=\hat{x}-\frac{3}{4}\left(\frac{|\hat{x}|}{k_{s} d}\right)^{2} \hat{x}=k_{s} x\left[1-\left(\frac{3}{4}\right)^{4}\left(\frac{x}{d}\right)^{8}\right]$

In this case, because the displacement of vibration is usually less than $10 \%$ of the gap, it is clear the nonlinear distortion is negligible after correction. In practice, it is difficult to apply the exact amount of nonlinearity correction as the exact value of $k_{S} d$ is not known. Spectrum analysis of the vibration signal may be used to predict the ratio between the displacement of vibration and the electrode gap.

\section{E. Nonlinear Dynamic Model}

By including stiffness and damping nonlinearities from the electrostatic drive and feedback controls described by equation (5), (14) and (15), the nonlinear dynamic model of a MEMS RIG with closed-loop feedback controls can be described by the differential equations of motion as:

$$
\begin{aligned}
{\left[\begin{array}{l}
\ddot{x} \\
\ddot{y}
\end{array}\right]+(\mathbf{C}+\mathbf{G})\left[\begin{array}{l}
\dot{x} \\
\dot{y}
\end{array}\right]+\mathbf{K}\left[\begin{array}{l}
x \\
y
\end{array}\right]=\left[\begin{array}{c}
\alpha y+\beta \dot{x} \\
-\alpha x+\beta \dot{y}
\end{array}\right]+} \\
{\left[\begin{array}{l}
k_{3} x^{3}+\frac{\alpha}{d^{2}} y^{3}+\frac{3 \beta}{d^{2}} x^{2} \dot{x} \\
k_{3} y^{3}-\frac{\alpha}{d^{2}} x^{3}+\frac{3 \beta}{d^{2}} y^{2} \dot{y}
\end{array}\right] }
\end{aligned}
$$

Here $\mathbf{C}=2 \vartheta_{0} \omega_{0}\left[\begin{array}{cc}1+\gamma_{1} & \gamma_{2} \\ \gamma_{2} & 1-\gamma_{1}\end{array}\right], \quad \mathbf{G}=\left[\begin{array}{cc}0 & -2 \Omega \\ 2 \Omega & 0\end{array}\right]$, $\mathbf{K}=\omega_{0}{ }^{2}\left[\begin{array}{cc}1+\mu_{1} & \mu_{2} \\ \mu_{2} & 1-\mu_{1}\end{array}\right]$, and $\alpha, \beta$ are automatically adjusted control gains, respectively used to maintain a constant amplitude of the major axis, and to null the minor axis in the elliptic coordinate system. Note that the linear stiffness softening term $\frac{C_{0} V_{d c}{ }^{2}}{d^{2}} x$ in equation (5) is seen as part of the entire linear stiffness $\mathbf{K}$. The quadratic term in equation (5) is neglected in the analysis as it only introduces a high frequency modulation of stiffness which is integrated to zero by control 
system. $k_{3}=\frac{2 C_{0} V_{d c}{ }^{2}}{d^{4} m}$ is the coefficient of electrostatic cubic nonlinearity. The second items at the right side of (18) are additional electrostatic nonlinearities to the linear RIG system.

\section{AVERAGED MOTION EQUATION}

\section{A. Linear Averaged Model}

The analysis of motion of the RIG is more convenient by expressing the equations of motion in the elliptic coordinate system in terms of amplitude and orbit phase. Transformation of the linear part of the equations of motion of the RIG system from the rectangular coordinates to the time averaged elliptical coordinates has been described in detail in [1][2]. The elliptic coordinate system is illustrated in figure (2) and the associated coordinate transformation equations is given by:

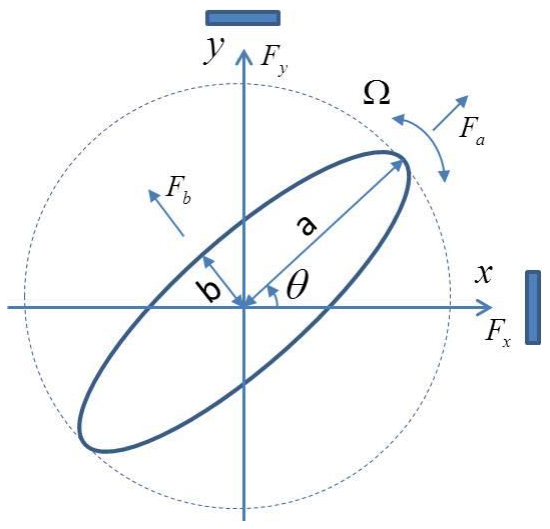

Fig. 2. The general elliptic orbit and precession of two-dimensional oscillator.

$\left\{\begin{array}{l}x(t)=a \cos \theta \cos (\omega t+\varphi)-b \sin \theta \sin (\omega t+\varphi) \\ y(t)=a \sin \theta \cos (\omega t+\varphi)+b \cos \theta \sin (\omega t+\varphi) \\ \dot{x}(t)=\omega(-a \cos \theta \sin (\omega t+\varphi)-b \sin \theta \cos (\omega t+\varphi)) \\ \dot{y}(t)=\omega(-a \sin \theta \sin (\omega t+\varphi)+b \cos \theta \cos (\omega t+\varphi))\end{array}\right.$

$\left[\begin{array}{llll}x & y & \dot{x} & \dot{y}\end{array}\right]^{T}$ are the fast time-varying state variables of displacement and velocity of vibration of the gyroscope's two second-order differential equations dynamic model. In the elliptic rotating coordinate system, the state variables are defined as slow time-varying $\left[\begin{array}{llll}a & b & \theta & \varphi\end{array}\right]^{T}$ to describe the orbital elements. $a$ is the amplitude of the elliptic major axis, $b$ is the amplitude of the elliptic minor axis, $\theta$ is the precession angle and $\varphi$ denotes the phase shift between the resultant drive force $f_{a}$ and response component $a(t)$ in the major axis. For the simplified case with no the nonlinear effects, the equations of motion in the slowly varying orbiting parameters are given by [3][8]:

$$
\begin{aligned}
& \dot{a}=-a v_{0} \omega_{0}\left(1+\gamma_{1} \cos 2 \theta+\gamma_{2} \sin 2 \theta\right)- \\
& \frac{1}{2} b \omega_{0}\left(\mu_{1} \sin 2 \theta-\mu_{2} \cos 2 \theta\right)+f_{a} \\
& \dot{b}=-b v_{0} \omega_{0}\left(1-\gamma_{1} \cos 2 \theta-\gamma_{2} \sin 2 \theta\right)+ \\
& \frac{1}{2} a \omega_{0}\left(\mu_{1} \sin 2 \theta-\mu_{2} \cos 2 \theta\right)+f_{b}
\end{aligned}
$$

$$
\begin{aligned}
\dot{\theta}= & -\Omega_{z}+\frac{\mathrm{a}^{2}+\mathrm{b}^{2}}{\mathrm{a}^{2}-\mathrm{b}^{2}} v_{0} \omega_{0}\left(\gamma_{1} \sin 2 \theta-\gamma_{2} \cos 2 \theta\right)- \\
& \frac{\mathrm{ab}}{\mathrm{a}^{2}-\mathrm{b}^{2}} \omega_{0}\left(\mu_{1} \cos 2 \theta+\mu_{2} \sin 2 \theta\right)+f_{\theta} \\
\dot{\varphi}= & \frac{1}{2} \omega_{0} \frac{\mathrm{a}^{2}+\mathrm{b}^{2}}{\mathrm{a}^{2}-\mathrm{b}^{2}}\left(\mu_{1} \cos 2 \theta+\mu_{2} \sin 2 \theta\right)+ \\
& \frac{a b}{\mathrm{a}^{2}-\mathrm{b}^{2}} 2 v_{0} \omega_{0}\left(-\gamma_{1} \sin 2 \theta+\gamma_{2} \cos 2 \theta\right)+\omega_{0}- \\
& \omega+f_{\phi}
\end{aligned}
$$

$f_{a}, f_{b}, f_{\theta}, f_{\phi}$ are effective forces in the elliptic coordinate system. When the orbit phase is kept to $\varphi=-\frac{\pi}{2}$ by a phaselocked loop, these control forces are simplified as:

$$
\left\{\begin{array}{l}
f_{a}=\left(\frac{1}{2} a \beta-\frac{1}{2 \omega} b \alpha\right) \\
f_{b}=\left(\frac{1}{2} b \beta-\frac{1}{2 \omega} a \alpha\right) \\
f_{\theta}=0 \\
f_{\phi}=0
\end{array}\right.
$$

The objectives of forces $f_{a}$ and $f_{b}$ are to respectively sustain a constant amplitude $a$ of the major axis, and null vibration at the minor axis. It is clear that non-proportional damping coefficients $\gamma_{1}$ and $\gamma_{2}$ result in a $2 \theta$ dependent drift error. Damping imperfections can be kept to less than $3 \%$ for high precision MEMS gyroscopes. The influence of stiffness imperfections $\mu_{1}$ and $\mu_{2}$ is negligible since $b$ is nulled by force $f_{b}$. The impact of stiffness imperfections $\mu_{1}$ and $\mu_{2}$ on the control of the minor axis $b$, resonant frequency $\varphi$ is more significant. The effect of $\mu_{1}$ and $\mu_{2}$ can also be observed through the quadrature control gain $\alpha$.

\section{B. Nonlinear Effects}

The cubic nonlinear stiffness and damping terms as formulated in equations (18) affect the angular drift error as well as the feedback control required for the RIG. The influence is illustrated by the extra terms they add to the averaged motion equations. By using the transformation equation and applying the averaging operation to the terms $x^{2}$ and $y^{2}$, the diagonal nonlinear stiffness terms $\left[\begin{array}{ll}k_{3} x^{3} & k_{3} y^{3}\end{array}\right]^{T}$ originating from the electrostatic force are described as:

$$
\begin{aligned}
& \frac{k_{3}}{4}\left(\left(\left(a^{2}+b^{2}\right)+\left(a^{2}-b^{2}\right) \cos 2 \omega t\right) \boldsymbol{I}+\left(\left(a^{2}-\right.\right.\right. \\
& \left.\left.\left.b^{2}\right) \cos 2 \theta+\left(a^{2}+b^{2}\right) \cos 2 \theta \cos 2 \omega t\right) \boldsymbol{V}\right)\left[\begin{array}{l}
x \\
y
\end{array}\right]
\end{aligned}
$$

The off-diagonal stiffness nonlinearity terms $\left[\frac{\alpha}{d^{2}} y^{3}-\frac{\alpha}{d^{2}} x^{3}\right]^{T}$ introduced by the quadrature nulling control can be expressed as:

$$
\begin{aligned}
& \frac{\alpha}{4 d^{2}}\left(\left(\left(a^{2}+b^{2}\right)+\left(a^{2}-b^{2}\right) \cos 2 \omega t\right) \boldsymbol{U}-\left(\left(a^{2}-\right.\right.\right. \\
& \left.\left.\left.b^{2}\right) \cos 2 \theta+\left(a^{2}+b^{2}\right) \cos 2 \theta \cos 2 \omega t\right) \boldsymbol{W}\right)\left[\begin{array}{l}
x \\
y
\end{array}\right]
\end{aligned}
$$


Similarly, the nonlinear damping forces $\left[\frac{3 \beta}{d^{2}} x^{2} \dot{x}, \quad \dot{\theta}=\frac{a b}{2 \omega}\left(\frac{k_{3}}{2}+\frac{k_{3}}{4} \cos 4 \theta-\frac{\alpha}{4 d^{2}} \sin 4 \theta\right)-\frac{3 \beta}{32 d^{2}}\left(a^{2}+\right.\right.$ $\left.\frac{3 \beta}{d^{2}} y^{2} \dot{y}\right]^{T}$ introduced by energy sustain control are represented by:

$\frac{3 \beta}{4 d^{2}}\left(\left(\left(a^{2}+b^{2}\right)+\left(a^{2}-b^{2}\right) \cos 2 \omega t\right) \boldsymbol{I}+\left(\left(a^{2}-\right.\right.\right.$

$\left.\left.\left.b^{2}\right) \cos 2 \theta+\left(a^{2}+b^{2}\right) \cos 2 \theta \cos 2 \omega t\right) \boldsymbol{V}\right)\left[\begin{array}{l}\dot{x} \\ \dot{y}\end{array}\right]$

Here the four matrices are $\boldsymbol{I}=\left[\begin{array}{ll}1 & 0 \\ 0 & 1\end{array}\right], \boldsymbol{U}=\left[\begin{array}{cc}0 & 1 \\ -1 & 0\end{array}\right], \boldsymbol{V}=$ $\left[\begin{array}{cc}1 & 0 \\ 0 & -1\end{array}\right]$, and $\boldsymbol{W}=\left[\begin{array}{ll}0 & 1 \\ 1 & 0\end{array}\right]$, such that the stiffness and damping nonlinearities are represented in a tight form as in [1]:

$$
\left[\begin{array}{l}
N_{x} \\
N_{y}
\end{array}\right]=A\left[\begin{array}{l}
x \\
y
\end{array}\right]+B\left[\begin{array}{l}
\dot{x} \\
\dot{y}
\end{array}\right]
$$

$A$ and $B$ are represented in "quaternion form":

$$
\begin{aligned}
& A=\alpha_{1} \boldsymbol{I}+\alpha_{2} \boldsymbol{U}+\alpha_{3} \boldsymbol{V}+\alpha_{4} \boldsymbol{W} \\
& B=\beta_{1} \boldsymbol{I}+\beta_{2} \boldsymbol{U}+\beta_{3} \boldsymbol{V}+\beta_{4} \boldsymbol{W}
\end{aligned}
$$

The associated coefficients are:

$$
\left\{\begin{array}{l}
\alpha_{1}=\frac{k_{3}}{4}\left(\left(a^{2}+b^{2}\right)+\left(a^{2}-b^{2}\right) \cos 2 \omega t\right) \\
\alpha_{2}=\frac{\alpha}{4 d^{2}}\left(\left(a^{2}+b^{2}\right)+\left(a^{2}-b^{2}\right) \cos 2 \omega t\right) \\
\alpha_{3}=\frac{k_{3}}{4}\left(\left(a^{2}-b^{2}\right) \cos 2 \theta+\left(a^{2}+b^{2}\right) \cos 2 \theta \cos 2 \omega t\right) \\
\alpha_{4}=-\frac{\alpha}{4 d^{2}}\left(\left(a^{2}-b^{2}\right) \cos 2 \theta+\left(a^{2}+b^{2}\right) \cos 2 \theta \cos 2 \omega t\right)
\end{array}\right.
$$

$\left\{\begin{array}{l}\beta_{1}=\frac{3 \beta}{4 d^{2}}\left(\left(a^{2}+b^{2}\right)+\left(a^{2}-b^{2}\right) \cos 2 \omega t\right) \\ \beta_{2}=0 \\ \beta_{3}=\frac{3 \beta}{4 d^{2}}\left(\left(a^{2}-b^{2}\right) \cos 2 \theta+\left(a^{2}+b^{2}\right) \cos 2 \theta \cos 2 \omega t\right) \\ \beta_{4}=0\end{array}\right.$

The effect of nonlinearity on the averaged motion equations can be derived following the process detailed in [1] and applying averaging over one period to yield:

$$
\begin{aligned}
\dot{a}= & \frac{1}{2 \omega}\left[-\frac{\alpha b}{16 d^{2}}\left(a^{2}+9 b^{2}\right)+\frac{k_{3} b}{16}\left(a^{2}-3 b^{2}\right) \sin 4 \theta+\right. \\
& \left.\frac{\alpha b}{16 d^{2}}\left(a^{2}-3 b^{2}\right) \cos 4 \theta\right]+\frac{3 a \beta}{64 d^{2}}\left(5 a^{2}+9 b^{2}\right)+ \\
& \frac{3 a \beta}{64 d^{2}}\left(a^{2}-3 b^{2}\right) \cos 4 \theta \\
\dot{b}= & \frac{1}{2 \omega}\left[-\frac{3 \alpha a}{16 d^{2}}\left(3 a^{2}-b^{2}\right)-\frac{k_{3}}{16} a\left(3 a^{2}-b^{2}\right) \sin 4 \theta-\right. \\
& \left.\frac{\alpha a}{16 d^{2}}\left(3 a^{2}-b^{2}\right) \cos 4 \theta\right]+\frac{9 b \beta}{32 d^{2}}\left(a^{2}+b^{2}\right)- \\
& \frac{3 b \beta}{32 d^{2}}\left(3 a^{2}-b^{2}\right) \cos 4 \theta
\end{aligned}
$$

$$
\begin{aligned}
\dot{\varphi}= & \frac{\left(a^{2}+b^{2}\right)}{2 \omega}\left[-\frac{9 k_{3}}{16}-\frac{3 k_{3}}{16} \cos 4 \theta+\frac{3 \alpha}{16 d^{2}} \sin 4 \theta\right]+ \\
& \frac{3 a b \beta}{8 d^{2}} \sin 4 \theta
\end{aligned}
$$

Dynamic equations (33) to (36) describe the influence of nonlinearities on all four state variables of the angular procession of RIG in the elliptic frame. Equations (33) to (36) can be simplified by ignoring terms that involves the amplitude of the minor axis $b$ as it is nulled by the quadrature loop. It is clear that effects of stiffness nonlinearity on drift error can be readily eliminated by quad nulling. Angular drift error is dominated by damping nonlinearities and imperfections. Equation (35) shows that the $4 \theta$ component to angular drift increases with control gain $\beta$. This highlights the benefit of having a high inherent $Q$ factor, as in the HRG, since the required gain $\beta$ for energy sustain control is reduced. Equation (36) illustrates the existence of a softening offset to the resonance frequency caused by electrostatic drive force, as well as a $4 \theta$ harmonic contribution.

\section{SIMULATION}

The MEMS RIG including the associated digital feedback control system is simulated with MATLAB/Simulink to verify the influence of electrostatic nonlinearities predicted in the theoretical analysis. The dynamic model is represented in Simulink block diagram as shown in figure (3). Nonlinearities are preserved in the electrostatic excitation and capacitive displacement measurement by using original nonlinear equations for the electrostatic drive force $F_{e}=\frac{\varepsilon A}{2(d-x)^{2}}\left(V_{d c}-\right.$ $\left.V_{a c}\right)^{2}$, and for the capacitive vibration detection $V_{\text {out }}=$ $\frac{1}{C_{F}} \frac{\varepsilon A}{d-x} V_{d c}$. The parameters used in the simulation correspond to the practical interface circuit, where the DC bias voltage is set at 25 volts, resonator mass $16.5 \mathrm{e}-7$, and feedback capacitor $\mathrm{Cf}$ of $5 \mathrm{pF}$. The gyroscope used in this investigation has an electrode gap of 10um, the non-proportional dampings are about $\gamma_{1}=0.02$ and $\gamma_{2}=0.006$, and the residual stiffness imperfections after mode tuning are about $\mu_{1}=-1 e^{-6}$ and $\mu_{2}=2 e^{-6}$, corresponding to a frequency split of about 20 $\mathrm{mHz}$. 


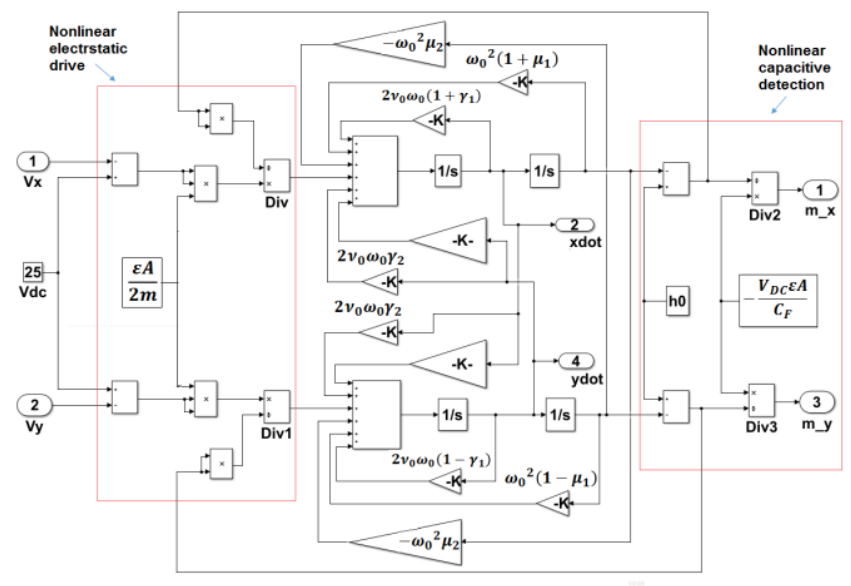

Fig. 3. Simulink block diagram of CVG dynamics including nonlinearities from transducer.

The entire RIG system is shown in figure (4). It includes digitization and FIR low pass filtering, digital orthogonal demodulation to calculate the in-phase and quadrature components for each mode of vibrations. The demodulation results $C_{x}, S_{x}, C_{y}, S_{y}$ are then transformed into the elliptic coordinate system to obtain elliptic parameters: amplitude of elliptic major axis $a$, minor axis $b$, angular location of the standing wave $\theta$, and the orbit phase $\varphi$. These elliptic parameters are thereafter used in the PLL resonance frequency tracking, energy sustain and quadrature nulling feedback control loops to fulfil the entire RIG measurement.

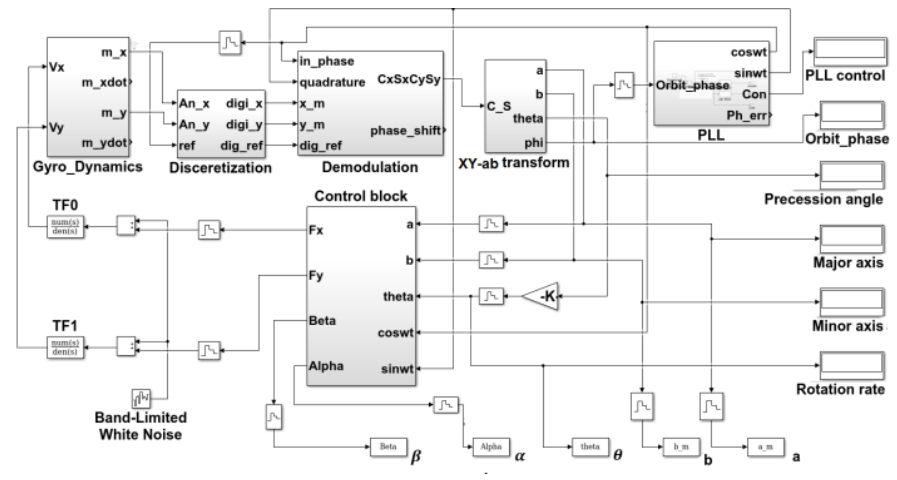

Fig. 4. Simulink block diagram of a RIG with closed-loop feedback control.

\section{A. Quadrature}

Equation (34) describes the nonlinear behavior of the quadrature control. The cubic stiffness nonlinearity from electrostatic drive has a large coefficient $k_{3}$ and it is not attenuated by the nulled minor axis $b$ as for the energy sustain control. This make quadrature control gain $\alpha$ the most noticeable nonlinear behavior even at a rather low amplitude of vibration. When the amplitude of major axis is set to $a=0.2$, it is equivalent to about $10 \%$ of the capacitive gap. Figure (5) shows the simulation results when varying the amplitude of vibration. As expected, the $4 \theta$ harmonics increase as the amplitude of the major axis is increased. The $2 \theta$ harmonics remains unchanged. Simulations also confirm that the nonlinear correction proposed in equation (17) has no effect on reducing $4 \theta$ harmonics, which is caused by nonlinear electrostatic drive.
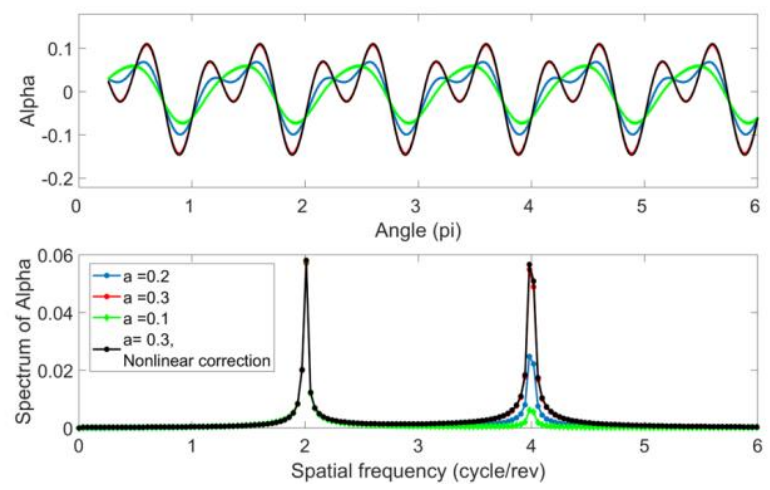

Fig. 5. Nonlinear behavior seen on the quadrature control as amplitude of vibration increases.

\section{B. Angular Drift Error}

As described in equation (35), the $4 \theta$ harmonics in the angular drift error is caused by damping nonlinearity introduced by velocity feedback to compensate the energy loss due to average damping. This high order drift error is proportionally related to the control gain $\beta$, as well as the ratio between the amplitude of vibration and the capacitive gap.
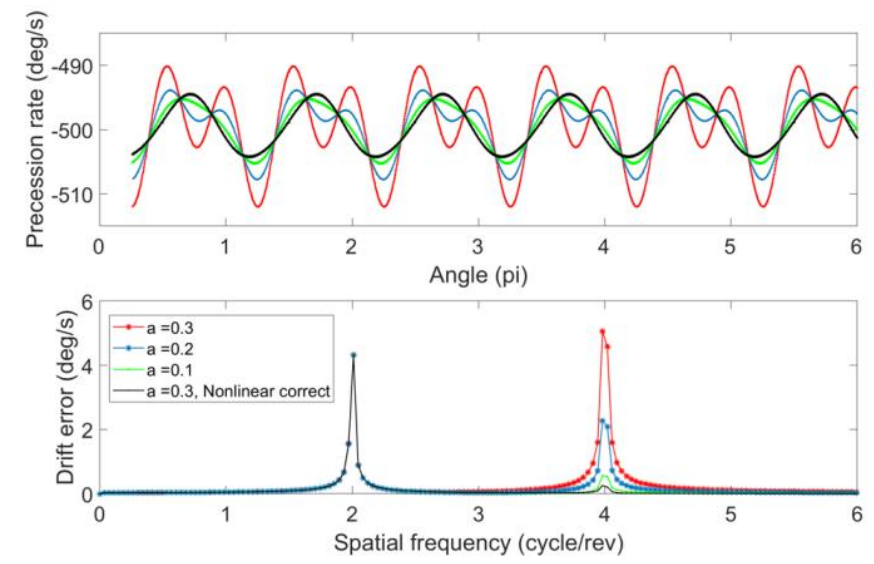

Fig. 6. $4 \theta$ harmonic drift error increases with major axis $a$, it is removed by nonlinear correction.

Figure (6) shows the angle dependent drift error. The $2 \theta$ harmonic is determined by the linear damping imperfections $\gamma_{1}, \gamma_{2}$. It remains constant as the amplitude of the major axis increases, while the $4 \theta$ harmonic drift error grows dramatically. Nonlinear correction described in (17) reduces the $4 \theta$ harmonic from $5 \mathrm{deg} / \mathrm{s}$ to $0.2 \mathrm{deg} / \mathrm{s}$ for major axis of 0.3 . The small residual $4 \theta$ harmonic error is caused by neglected high order nonlinear terms in equation (7) of the capacitive detection.

\section{Resonance Frequency}

Fluctuation of resonance frequency is the result of stiffness imperfections $\mu_{1}$ and $\mu_{2}$, and the nonlinearity from electrostatic drive and quadrature control. Linear stiffness imperfections $\mu_{1}$ and $\mu_{2}$ lead to the 2 th angular modulation of the resonance frequency. Nonlinearity causes $4 \theta$ angular modulation of the frequency and a frequency softening offset, as indicated by equation (36). Simulation results in figure 7 shows an 
increasing frequency offset and the 4th harmonics as the amplitude of vibration increases. The $2 \theta$ harmonics remains constant. Nonlinear correction of the capacitive detection shows no effect on the resonance frequency as expected in the analysis.
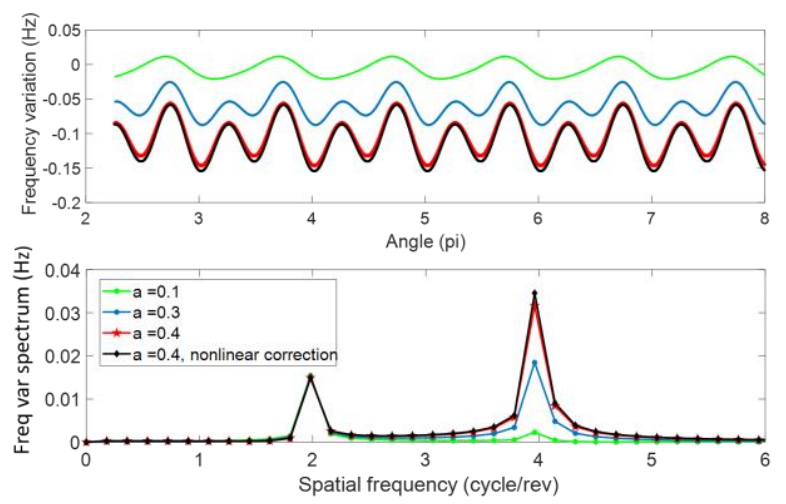

Fig. 7. Affected by nonlinear electrostatic force, tracked resonance frequency shows a $4^{\text {th }}$ angular modulation and an offset increasing with amplitude of vibration.

\section{EXPERIMENTAL RESULTS}

This section describes the closed-loop control necessary for the operation of the MEMS rate integrating gyroscope, and the experimental tests conducted to investigate the nonlinear effects on sensor performance and real time control of the system. The experimental setup is shown in figure (8), which is capable of RIG testing with an external continuous rate input up to 300 rpm.

The vibrating structure of the gyroscope is a suspended ring, which has a radius of $4 \mathrm{~mm}$, width of $200 \mu \mathrm{m}$ and thickness of $150 \mu \mathrm{m}$. The capacitive gap formed between the ring and the electrodes is $10 \mu \mathrm{m}$. The device is fabricated from silicon $<111>$ by Silicon Sensing. Details of electrode arrangement of the drive, sense and mode tuning can be found in previous reports [19]. Figure 8.b shows the vacuum packaged MEMS gyroscope device.
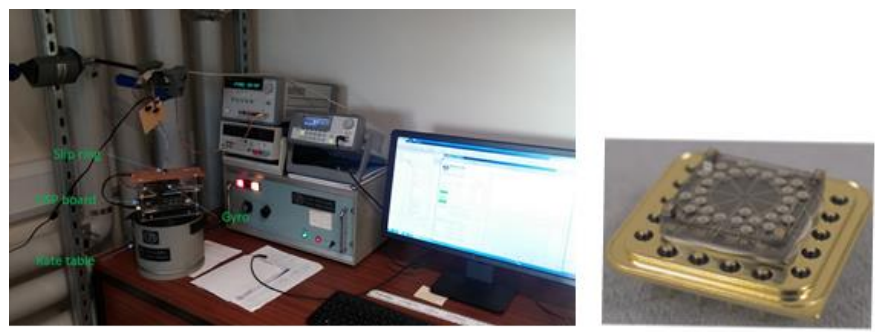

Fig. 8. Experimental setup, and the packaged MEMS ring gyroscope.

\section{A. Control platform}

Closed-loop control of the rate integrating gyroscope is incorporated onto a DSP based gyroscope control system. The system block diagram is shown in figure 9. The signal generation is based on an audio codec chip with $192 \mathrm{KHz}$ sampling rate, and the principle of direct digital synthesis technique (DDS) is used to create high quality sine/cosine waves for resonance excitations. The frequency resolution is $0.3 \mathrm{mHz}$. It also provides the digital reference for orthogonal synchronous detection. The digital orthogonal demodulation plays a key role to resolve the in-phase and quadrature components of the detected vibrations signals, which are used to calculate the amplitude and phase information of both the primary and sense modes for real time dynamic control.

The control for RIG consists of five major modules: (1) Vibration detection via digital orthogonal demodulation, which provides the quadrature and in-phase components of vibration $S_{x}, C_{x}, S_{y}, C_{y}$; (2) Resonance frequency tracking based on a PLL that tracks the resonance frequency; (3) Energy sustain by velocity feedback to maintain a constant amplitude of the major axis of the elliptic trajectory; (4) Quad nulling based on an anti-skew displacement feedback to supress the minor axis without disturbing angular precession. (5) State feedback to compensate damping and stiffness imperfections, details of feedback compensation can be found in [4][8]. System states, the velocity and displacement, for feedback control are recreated using the demodulation results $C_{x}, S_{x}, C_{y}, S_{y}$ and the digital signal reference.

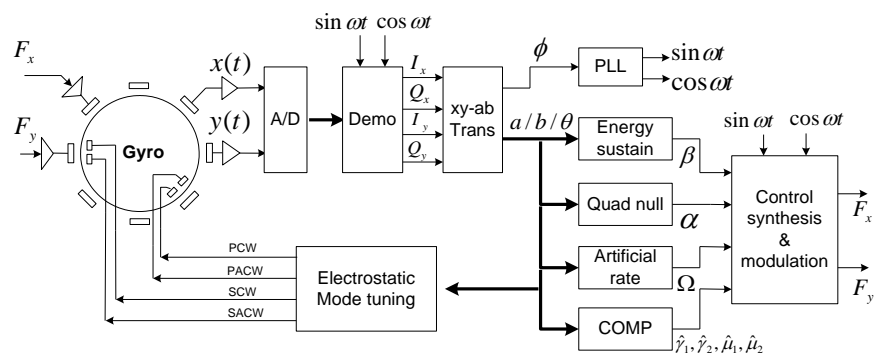

Fig. 9. Control system block diagram for the MEMS rate integrating gyroscope.

\section{B. Rate Table Testing}

Based on the DSP controlled RIG system, a series of practical rate table testing were conducted to validate the theoretical analysis of the electrostatic nonlinearity, and the effectiveness of the nonlinear correction proposed for reducing the high order drift error. In real time closed-loop control of the RIG, quadrature control demonstrates obvious nonlinear behavior, while the nonlinear influence on the angular precession needs spectrum analysis to show the harmonics. Figure 10 plots the nonlinear behavior of the quadrature control that increases with the amplitude of the major axis.

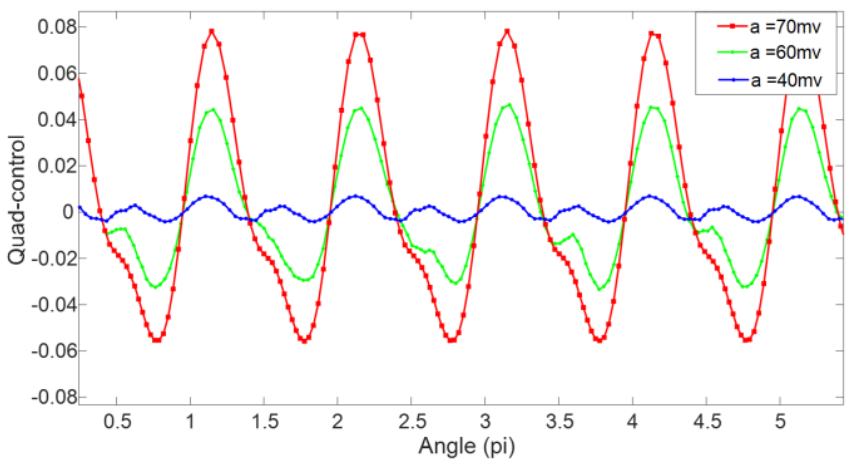

Fig. 10. Nonlinear behavior of the quadrature control that increases with amplitude of the major axis. 
Figure 11 shows the test results for an external continuous input rate of $28 \mathrm{deg} / \mathrm{s}$, and amplitude of the major axis is set to $30 \mathrm{mv}$. Without velocity feedback compensation to cancel the linear non-proportional damping $\gamma_{1}, \gamma_{2}$, the sensor output shows a high angle dependent drift error, the amplitude of periodic fluctuation of the drift rate is about $3.5 \mathrm{deg} / \mathrm{s}$. With the aid of parameter estimation of $\gamma_{1}, \gamma_{2}$ and applying velocity feedback compensation [3], the $2 \theta$ drift error is reduced to 0.15 $\mathrm{deg} / \mathrm{s}$ whilst the $4 \theta$ drift remains almost unaffected. When the nonlinear correction is applied, and by carefully adjusting correction parameter $k_{s} d$ the $4 \theta$ harmonic drift error is further reduced to $0.16 \mathrm{deg} / \mathrm{s}$.
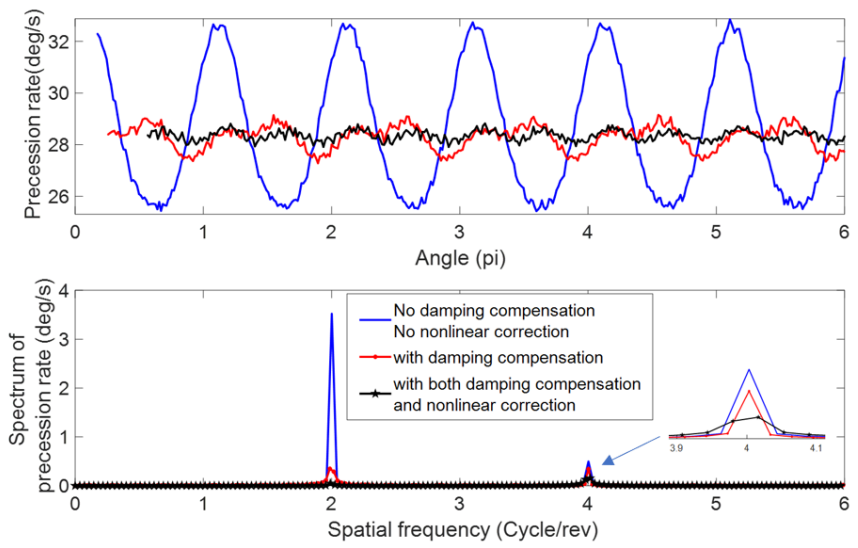

Fig.11. Angualr drift error is reduced to less than $0.3 \mathrm{deg} / \mathrm{s}$ by feedback compensation and nonlinear correction.

\section{CONCLUSION}

This paper presents analysis and formulation of the influence of nonlinearities on the drift error performance and real time control of an electrostatic MEMS ring type rate integrating gyroscope, and proposes a nonlinear correction to reduce the $4 \theta$ harmonic drift error. As the gyroscope's ring resonant structure is relatively large, and the device has extremely low damping, nonlinearities originate from electrostatic forces and capacitive sensing dominate. Nonlinearities originate from geometrical and material characteristics are neglected in the analysis. The analysis shows that stiffness nonlinearities enter into the dynamic system directly via electrostatic force and quadrature control, and that damping nonlinearity is introduced by state feedback for energy sustaining control. It is demonstrated by the averaged motion equations of the nonlinear RIG in the elliptic coordinate system that stiffness nonlinearities result in $4 \theta$ harmonics on the quadrature control and resonance frequency tracking, and that damping nonlinearity causes a $4 \theta$ harmonic drift error. Validation of the theoretical analysis and effectiveness of the nonlinear correction is provided by full simulation of the digitally closed-loop controlled RIG. Rate table experimental results show drift error less than $0.2 \mathrm{deg} / \mathrm{s}$ can be achieved by feedback compensation of non-ideal damping and the proposed nonlinear correction

\section{APPENDIX}

For the convenience of readers, this appendix provides a brief description of the formulation of the averaged motion equation of the RIG in orbital parameters in the rotating elliptic coordinate system, which is derived in great details by Bernard in [1]. The motion equations (18) of CVGs in the Cartesian coordinate system can be reformatted as

$$
\begin{aligned}
& {\left[\begin{array}{c}
\ddot{x} \\
\ddot{y}
\end{array}\right]+\mathbf{G}\left[\begin{array}{c}
\dot{x} \\
\dot{y}
\end{array}\right]+\omega_{0}{ }^{2}\left[\begin{array}{l}
x \\
y
\end{array}\right]=\mathbf{C}\left[\begin{array}{l}
\dot{x} \\
\dot{y}
\end{array}\right]+\left[\begin{array}{cc}
\omega_{0}{ }^{2} \mu_{1} & \omega_{0}{ }^{2} \mu_{2} \\
\omega_{0}{ }^{2} \mu_{2} & -\omega_{0}{ }^{2} \mu_{1}
\end{array}\right]\left[\begin{array}{l}
x \\
y
\end{array}\right]+} \\
& {\left[\begin{array}{c}
\alpha y+\beta \dot{x} \\
-\alpha x+\beta \dot{y}
\end{array}\right]+\left[\begin{array}{l}
k_{3} x^{3}+\frac{\alpha}{d^{2}} y^{3}+\frac{3 \beta}{d^{2}} x^{2} \dot{x} \\
k_{3} y^{3}-\frac{\alpha}{d^{2}} x^{3}+\frac{3 \beta}{d^{2}} y^{2} \dot{y}
\end{array}\right]}
\end{aligned}
$$

The terms on the right-hand side represent perturbations to the motion equations of an ideal gyroscope that are potential sources of error in practical devices. They can be formulated as a two-component "perturbation vector":

$$
\left[\begin{array}{l}
p_{x} \\
p_{y}
\end{array}\right]=\boldsymbol{A}\left[\begin{array}{l}
x \\
y
\end{array}\right]+\boldsymbol{B}\left[\begin{array}{l}
\dot{x} \\
\dot{y}
\end{array}\right]
$$

Where $\boldsymbol{A}$ and $\boldsymbol{B}$ are represented in the form of "quaternion vector":

$$
\begin{aligned}
& \boldsymbol{A}=\alpha_{1} \boldsymbol{I}+\alpha_{2} \boldsymbol{U}+\alpha_{3} \boldsymbol{V}+\alpha_{4} \boldsymbol{W} \\
& \boldsymbol{B}=\beta_{1} \boldsymbol{I}+\beta_{2} \boldsymbol{U}+\beta_{3} \boldsymbol{V}+\beta_{4} \boldsymbol{W}
\end{aligned}
$$

Where the four matrices are

$$
\boldsymbol{I}=\left[\begin{array}{ll}
1 & 0 \\
0 & 1
\end{array}\right], \boldsymbol{U}=\left[\begin{array}{cc}
0 & 1 \\
-1 & 0
\end{array}\right], \boldsymbol{V}=\left[\begin{array}{cc}
1 & 0 \\
0 & -1
\end{array}\right], \boldsymbol{W}=\left[\begin{array}{ll}
0 & 1 \\
1 & 0
\end{array}\right]
$$

Using the transformation defined in (19), the differential equations in the orbital elements is given by:

$$
\begin{aligned}
\dot{a}= & \frac{1}{2 \omega}\left\{-\alpha_{1} a \sin 2 \omega t-\alpha_{2} b(1-\cos 2 \omega t)+\right. \\
& \alpha_{3}[b \sin 2 \theta(1-\cos 2 \omega t)-a \cos 2 \theta \sin 2 \omega t]+ \\
& \left.\alpha_{4}[-\mathrm{b} \cos 2 \theta(1-\cos 2 \omega t)-a \sin 2 \theta \sin 2 \omega t]\right\}+ \\
& \frac{1}{2}\left\{\beta_{1} a(1-\cos 2 \omega t)-\beta_{2} b \sin 2 \omega t+\beta_{3}[a \cos 2 \theta(1-\right. \\
& \cos 2 \omega t)+b \sin 2 \theta \sin 2 \omega t]+\beta_{4}[a \sin 2 \theta(1- \\
& \cos 2 \omega t)-b \cos 2 \theta \sin 2 \omega t]\}
\end{aligned}
$$

$$
\begin{aligned}
\dot{b}= & \frac{1}{2 \omega}\left\{\alpha_{1} b \sin 2 \omega t-\alpha_{2} a(1+\cos 2 \omega t)+\right. \\
& \alpha_{3}[-a \sin 2 \theta(1+\cos 2 \omega t)-b \cos 2 \theta \sin 2 \omega t]+ \\
& \left.\alpha_{4}[\operatorname{acos} 2 \theta(1+\cos 2 \omega t)-b \sin 2 \theta \sin 2 \omega t]\right\}+ \\
& \frac{1}{2}\left\{\beta_{1} b(1+\cos 2 \omega t)+\beta_{2} a \sin 2 \omega t+\beta_{3}[-b \cos 2 \theta(1+\right. \\
& \cos 2 \omega t)+a \sin 2 \theta \sin 2 \omega t]+\beta_{4}[-b \sin 2 \theta(1+ \\
& \cos 2 \omega t)-a \cos 2 \theta \sin 2 \omega t]\}
\end{aligned}
$$

$$
\begin{aligned}
\dot{\theta}= & \frac{1}{2 \omega\left(a^{2}-b^{2}\right)}\left\{2 \alpha_{1} a b \cos 2 \omega t+\alpha_{2}\left(a^{2}+b^{2}\right) \sin 2 \omega t+\right. \\
& \alpha_{3}\left[2 a b \cos 2 \theta+\left(a^{2}-b^{2}\right) \sin 2 \theta \sin 2 \omega t\right]+ \\
& \left.\alpha_{4}\left[2 a b \sin 2 \theta-\left(a^{2}-b^{2}\right) \cos 2 \theta \sin 2 \omega t\right]\right\}+ \\
& \frac{1}{2\left(a^{2}-b^{2}\right)}\left\{-2 \beta_{1} a b \sin 2 \omega t+\beta_{2}\left[-\left(a^{2}-b^{2}\right)+\right.\right. \\
& \left.\left(a^{2}+b^{2}\right) \cos 2 \omega t\right]+\beta_{3}\left[-\left(a^{2}+b^{2}\right) \sin 2 \theta+\right. \\
& \left.\left(a^{2}-b^{2}\right) \sin 2 \theta \cos 2 \omega t\right]+\beta_{4}\left[\left(a^{2}+b^{2}\right) \cos 2 \theta-\right. \\
& \left.\left.\left(a^{2}-b^{2}\right) \cos 2 \theta \cos 2 \omega t\right]\right\}-\Omega
\end{aligned}
$$




$$
\begin{aligned}
\dot{\varphi}= & \frac{1}{2 \omega\left(\left(a^{2}-b^{2}\right)\right)}\left\{\alpha_{1}\left[-\left(a^{2}-b^{2}\right)-\left(a^{2}+b^{2}\right) \cos 2 \omega t\right]-\right. \\
& 2 \alpha_{2} a b \sin 2 \omega t+\alpha_{3}\left[-\left(a^{2}+b^{2}\right) \cos 2 \theta-\left(a^{2}-\right.\right. \\
& \left.\left.b^{2}\right) \cos 2 \theta \cos 2 \omega t\right]+\alpha_{4}\left[-\left(a^{2}+b^{2}\right) \sin 2 \theta-\left(a^{2}-\right.\right. \\
& \left.\left.\left.b^{2}\right) \sin 2 \theta \cos 2 \omega t\right]\right\}+\frac{1}{2\left(a^{2}-b^{2}\right)}\left\{\beta_{1}\left(a^{2}+b^{2}\right) \sin 2 \omega t-\right. \\
& 2 \beta_{2} a b \cos 2 \omega t+\beta_{3}\left[2 a b \sin 2 \theta+\left(a^{2}-\right.\right. \\
& \left.\left.b^{2}\right) \cos 2 \theta \sin 2 \omega t\right]+\beta_{4}\left[-2 a b \cos 2 \theta+\left(a^{2}-\right.\right. \\
& \left.\left.\left.b^{2}\right) \sin 2 \theta \sin 2 \omega t\right]\right\}+\omega
\end{aligned}
$$

Averaged motion equations can be obtained by averaging the periodic terms of $\omega t$, which is equivalent to deleting all terms containing $\sin 2 \omega t, \cos 2 \omega t$, and $\sin 4 \omega t, \cos 4 \omega t$ when nonlinearity terms are involved in the dynamics.

\section{REFERENCES}

[1]. Bernard Friedland and M.F. Hutton, "Theory and error analysis of vibrating-member gyroscope," IEEE Trans. Automatic Control, vol. AC-23, pp.545-556, Aug. 1978.

[2]. D.D. Lynch, "Vibratory Gyro Analysis by the Method of Averaging," International conference on Gyroscopic Technology and Navigation, St. Petersburg, 1995.

[3]. Zhongxu Hu, and Barry Gallacher, "Extended Kalman filtering based parameter estimation and drift compensation for a MEMS rate integrating gyroscope." Sensors and Actuators A: Physical, vol. 250, p96-105, Oct. 2016.

[4]. Zhongxu Hu, and Barry J Gallacher, "Precision mode tuning towards a low angle drift MEMS rate integrating gyroscope," Mechatronics, April 2017, in press.

[5]. Igor P. Prikhodko, Jeffrey A. Gregory, Dmitry I. Bugrov and Michael W. Judy, "Overcoming Limitations of Rate Integrating Gyroscopes by Virtual Rotation". International Symposium on Inertial Sensors and Systems Proceedings, CA, USA, Feb. 2016.

[6]. David D. Lynch, "MRIG frequency mismatch and quadrature control," International Symposium on Inertial Sensors and Systems Proceedings, CA, USA, 2014.

[7]. J. A. Gregory, J. Cho, and K. Najafi, "Novel mismatch compensation methods for rate-integrating gyroscopes," Position, Location and Navigation Symposium - PLANS, 2014 IEEE/ION, pp.252-258, April 2012.

[8]. Zhongxu Hu and B J Gallacher, "Control and damping imperfection compensation for a rate integrating MEMS gyroscope," DGON Inertial Sensors and Systems, Karlsruhe, Germany, pp.1-15, Sep. 2015.

[9]. Pavel M. Polunin, Yushi Yang, Mark I. Dykman, Thomas W. Kenny, and Steven W. Shaw. "Characterization of MEMS Resonator Nonlinearities Using the Ring down Response," J. Microelectromech. Syst, vol.25, pp297-303, April 2016.

[10].H. K. Lee, R. Melamud, S. Chandorkar, J. Salvia, S. Yoneoka, and T. W. Kenny, "Stable operation of MEMS oscillators far above the critical vibration amplitude in the nonlinear regime," J. Microelectromech. Syst., vol. 20, pp.1228-1230, 2011.

[11].M.C. Mestroma, R.H.B. Fey, J.T.M. van Beekb, K.L. Phan b, H. Nijmeijer, "Modelling the dynamics of a MEMS resonator: Simulations and experiments". Sensors and Actuators A physical, vol. 142, pp306-315, March 2008.

[12].Deepak K. Agrawal, Jim Woodhouse, and Ashwin A. Seshia, "Modeling Nonlinearities in MEMS Oscillators," IEEE Transactions on Ultrasonics, Ferroelectrics, and Frequency Control, vol. 60, pp1646-1659, Aug. 2013.

[13].S. Zaitsev, O. Shtempluck, E. Buks, and O. Gottlieb, "Nonlinear damping in a micromechanical oscillator," Nonlinear Dyn., vol. 67, pp.859-883, April. 2011.

[14]. A. Eichler, J. Moser, J. Chaste, M. Zdrojek, I. Wilson-Rae, and A. Bachtold, "Nonlinear damping in mechanical resonators made from carbon nanotubes and graphene," Nature Nanotechnol., vol. 6, pp. 339-342, April 2011.

[15].T. Tsukamoto and S. Tanaka, "Virtually rotated MEMS whole angle gyroscope using independently controlled $\mathrm{CW} / \mathrm{CCW}$ oscillations," 2018 IEEE International Symposium on Inertial Sensors and Systems (INERTIAL), Moltrasio, 2018, pp. 1-4.

[16].P. Taheri-Tehrani, A. D. Challoner and D. A. Horsley, "Micromechanical Rate Integrating Gyroscope With AngleDependent Bias Compensation Using a Self-Precession Method," in IEEE Sensors Journal, vol. 18, no. 9, pp. 3533-3543, 1 May1, 2018.

[17].E. Tatar, T. Mukherjee, and G.K. Fedder, "Nonlinearity tuning and its effects on the performance of a MEMS gyroscope," IEEE Transducers 2015, Anchorage, Alaska, USA, June 21-25, 2015.

[18].S. H. Nitzan, P. Taheri-Tehrani, M. Defoort, S. Sonmezoglu and D. A. Horsley, "Countering the Effects of Nonlinearity in RateIntegrating Gyroscopes," in IEEE Sensors Journal, vol. 16, no. 10, pp. 3556-3563, May15, 2016.

[19].P Taheri-Tehrani, M Defoort and D A Horsley, "Operation of a high quality-factor gyroscope in electromechanical nonlinearities regime," J. Micromech. Microeng, vol. 27, June 2017.

[20].S. Nitzan, T-H. Su, C. Ahn, E. Ng, V. Hong, Y. Yang, T. Kenny, and D.A. Horsley, "Impact of gyroscope operation above the critical bifurcation threshold on scale factor and bias instability", IEEE international conference on Micro Electro Mechanical Systems, CA, USA, 2014.

[21]. Barry Gallacher, "Principles of a micro rate integrating ring gyroscope," IEEE Transactions on Aerospace and electronics systems, vol. 48, pp658-672, Jan. 2012.

[22].Zhongxu Hu, Barry J Gallacher, JS Burdess, "A digital signal processing-based control system for a micro-electromechanical system vibrating gyroscope with parametric amplification and force rebalance control," Proceedings of the Institute of Mechanical engineers, Part I: Journal of systems and control engineering, vol 227, pp347-362, March 2013.

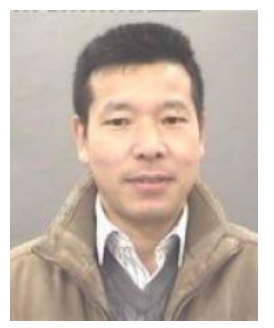

Zhongxu Hu is a senior research fellow in the Department of Mechanical \& System Engineering, Newcastle University upon Tyne, UK. He received his $\mathrm{PhD}$ at South China University of Technology in control theory and application in 2001. His research interests are in the fields of MEMS sensor dynamics, modelling and simulation, characterization, interface electronics, signal processing and advanced control system, especially MEMS inertial sensors and applications. Currently, He is working on attitude determination and control system for pico-satellites in Glasgow university.

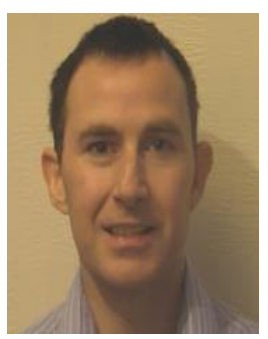

Barry J. Gallacher received the BSc degree in physics from the University of Glasgow in 1994. He received the $\mathrm{PhD}$ degree from the University of newcastle in 2002 for his work on vibratory gyroscopes. Between 1994 and 1999 he worked within the microelectronics industry, first with GEC Plessey Semiconductors and secondly with Siemens Microelectronics on thin film processing and characterization. He currently holds the position of Reader in Engineering Dynamics within the School of Mechanical and Systems Engineering where his current research interests encompass several areas within the field of microelectromechanical systems including the design of physical sensors (including inertial, viscosimetry and strain), non-linear dynamics, parametric excitation, control, reliability and microrefrigeration. 
